\title{
Magnetic lattices for ultracold atoms and degenerate quantum gases
}

\author{
Yibo Wang • Prince Surendran • Smitha Jose • \\ Tien Tran • Ivan Herrera • Shannon Whitlock • \\ Russell McLean • Andrei Sidorov • Peter Hannaford
}

\begin{abstract}
We review recent developments in the use of magnetic lattices as a complementary tool to optical lattices for trapping periodic arrays of ultracold atoms and degenerate quantum gases. Recent advances include the realisation of Bose-Einstein condensation in multiple sites of a magnetic lattice of one-dimensional microtraps, the trapping of ultracold atoms in square and triangular magnetic lattices, and the fabrication of magnetic lattice structures with sub-micron period suitable for quantum tunnelling experiments. Finally, we describe a proposal to utilise long-range interacting Rydberg atoms in a large spacing magnetic lattice to create interactions between atoms on neighbouring sites.
\end{abstract}

Keywords Magnetic lattices, ultracold atoms, degenerate quantum gases, quantum simulation

Yibo Wang, Prince Surendran, Smitha Jose, Tien Tran, Russell McLean, Andrei Sidorov, Peter Hannaford Centre for Quantum and Optical Science, Swinburne University of Technology, Melbourne, Victoria 3122, Australia

Ivan Herrera

Dodd-Walls Centre for Photonic and Quantum Technologies Department of Physics, University of Auckland,

Private Bag 92019, Auckland, New Zealand

Shannon Whitlock

Physikalisches Institut, Universität Heidelberg, Im Neuenheimer Feld 226, 69120 Heidelberg, Germany

P. Hannaford

email: phannaford@swin.edu.au

\section{Introduction}

Since the advent of laser cooling and trapping techniques in the 1980s and 90s [1-3], optical lattices produced by interfering laser beams have become an indispensable tool for trapping periodic arrays of ultracold atoms and degenerate quantum gases (see, e.g., [4-6] for reviews). Applications include quantum simulations of condensed matter phenomena [6], trapping of atom arrays in high precision atomic clocks [7] and quantum gas microscopes [8], and the realisation of quantum gates for quantum information processing $[9,10]$. These 'artificial crystals' allow precise control over system parameters, such as the lattice geometry, inter-particle interaction and lattice perfection, and, in principle, provide an ideal platform to achieve almost perfect realisations of a variety of condensed matter phenomena (e.g., [5, 6]). Examples include the superfluid to Mott insulator transition [11], the metal to insulator cross-over in honeycomb lattices [12], the Ising spin model for a 1D spin chain [13], the Hubbard model involving antiferromagnetic correlations [14], lowdimensional quantum systems $[15,16]$, disordered systems involving Anderson localisation [17, 18], topological edge states and the quantum Hall effect [19], and arrays of Josephson junctions [20].

An alternative approach for producing periodic lattices for trapping ultracold atoms involves the use of arrays of magnetic microtraps created by patterned magnetic films [21-36], current-carrying conductors [37-40], nanomagnetic domain walls [41], vortex arrays in superconducting films [42] or pulsed gradient magnetic fields $[43,44]$. In the present mini-review we focus on recent developments in the trapping of ultracold atoms in magnetic lattices of microtraps based on patterned magnetic films. These magnetic lattices have a high degree of design flexibility and may, in principle, be tailored with nearly arbitrary configurations and lattice spacing [29] without restrictions imposed by optical wavelengths. In addition, magnetic lattices do not require (intense) laser beams, they are free of spontaneous emission, they have relatively little technical noise or heating, and they involve state-selective atom trapping allowing radio-frequency (RF) evaporative cooling to be performed in the lattice and RF spectroscopy to be used to characterise the trapped atoms in situ [45, 46]. Finally, magnetic lattices are well suited for mounting on atom chips and incorporating into devices such as 'atomtronic' circuits [47]. However, magnetic lattices are still in their infancy compared with optical lattices, due largely to the difficulty of fabricating suitable magnetic microstructures with well controlled potentials, especially lattices with sub-micron periods suitable for quantum tunnelling. 
(a)

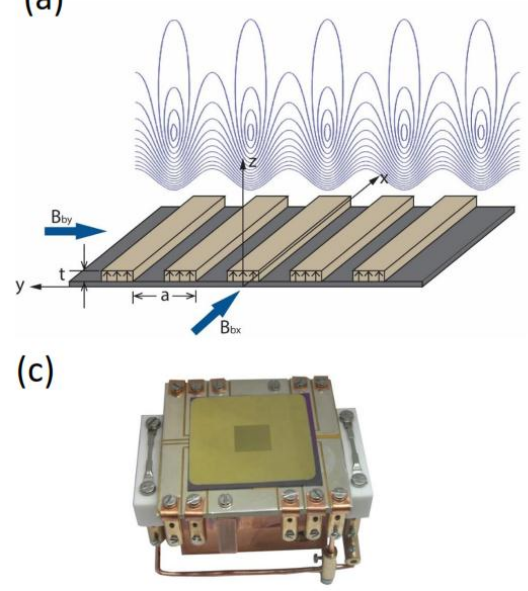

(b)

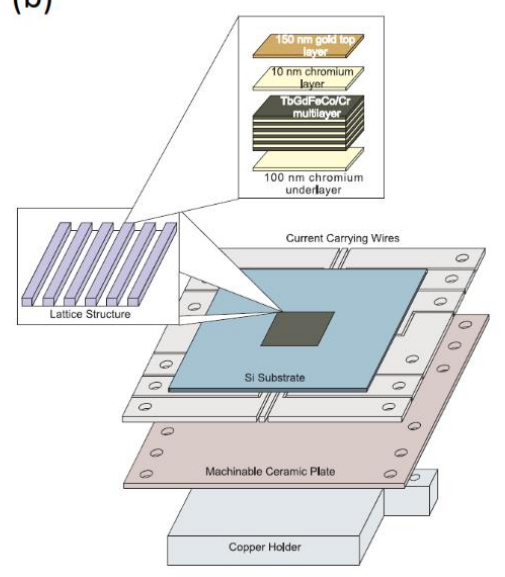

Fig. 1 (a) Magnetic lattice of 1D microtraps produced by an array of perpendicularly magnetised magnets with period $a$ and bias fields $B_{b x}, B_{b y}$ [58]. The contour lines are magnetic equipotentials calculated for typical parameters. (b) Construction of the magnetic lattice atom chip. The machined silver foil beneath the magnetic lattice structure contains U-shaped and Z-shaped current-carrying conductors for trapping and loading the atoms. Reproduced from Singh et al. [26] with the permission of IOP Publishing. (c) Photograph of the magnetic lattice atom chip coated with a reflecting gold film [59]

In this article we review recent progress in the development of magnetic lattices based on patterned magnetic films for trapping periodic arrays of ultracold atoms and degenerate quantum gases, and discuss future prospects for the application of magnetic lattices.

\section{One-dimensional magnetic lattices}

Magnetic lattices consisting of arrays of one-dimensional microtraps are a useful testing ground prior to progressing to more complex two-dimensional geometries. They are a natural extension of the magnetic mirrors [48] proposed by Opat et al. in 1992 [49] and subsequently realised using arrays of permanent magnets [50-52] and current-carrying conductors [53-55]. A magnetic mirror may be turned into a magnetic lattice of 1D microtraps by applying a uniform bias field to interfere with the rotating magnetic field of the periodic array (Fig. 1(a)), as described by Eq (1) below.

For an infinite periodic array of long magnets in the $x-y$ plane with perpendicular magnetisation $M_{z}$, periodicity $a$ and bias fields $B_{b x}, B_{b y}$, the magnetic field components at distances $z \gg a / 2 \pi$ from the bottom of the magnets are given approximately by [22]

$$
\left[B_{x} ; B_{y} ; B_{z}\right] \approx\left[B_{b x} ; B_{0} \sin (k y) e^{-k z}+B_{b y} ; B_{0} \cos (k y) e^{-k z}\right]
$$

where $k=2 \pi / a, B_{0}=4 M_{z}\left(e^{k t}-1\right)$ is a characteristic surface magnetic field (in Gaussian units), and $t$ is the thickness of the magnets. The magnetic field minimum (or trap bottom) $B_{\text {min }}$, trapping height $z_{\text {min }}$, barrier heights $\Delta B_{y, z}$, and trap frequencies $\omega_{y, z}$ for an atom of mass $m$ in a harmonic trapping potential are given by

$$
\begin{aligned}
& B_{\text {min }}=\left|B_{b x}\right| \\
& z_{\text {min }}=\frac{a}{2 \pi} \ln \left(\frac{B_{0}}{\left|B_{b y}\right|}\right)
\end{aligned}
$$

$$
\begin{aligned}
& \Delta B_{y}=\left(B_{b x}^{2}+4 B_{b y}^{2}\right)^{\frac{1}{2}}-\left|B_{b x}\right| \\
& \Delta B_{z}=\left(B_{b x}^{2}+B_{b y}^{2}\right)^{\frac{1}{2}}-\left|B_{b x}\right| \\
& \omega_{y}=\omega_{z}=\omega_{r a d}=\frac{2 \pi}{a}\left(\frac{m_{F} g_{F} \mu_{B}}{m\left|B_{b x}\right|}\right)^{1 / 2}\left|B_{b y}\right|
\end{aligned}
$$

where $m_{F}$ is the magnetic quantum number, $g_{F}$ is the Landé g-factor and $\mu_{B}$ is the Bohr magneton. $B_{\text {min }}, z_{\text {min }}$, $\Delta B_{y, z}$ and $\omega_{y, z}$ may all be controlled by adjusting the bias fields $B_{b x}$ and $B_{b y}$. Equations (1) - (5) illustrate how the characteristics of the magnetic lattice can be varied by varying the bias fields $B_{b x}, B_{b y}$ and how the magnetic lattice can be switched on or off by switching $B_{b y}$ on or off. Equations (2) - (5) are useful for providing scalings for the various parameters.

In 2005 Sinclair et al. [56] created a periodic array of 1D magnetic traps, or magnetic 'waveguides', made from a sinusoidal magnetisation pattern of period $106 \mu \mathrm{m}$ written on videotape plus bias fields, and successfully produced a single Bose-Einstein condensate (BEC) in one of the waveguides. In 2007 Boyd et al. [57] created an array of 1D traps produced by a hard disk platter written with a periodic pattern of period $100 \mu \mathrm{m}$ plus bias fields, and produced a condensate in one of the traps.

In 2008 Singh et al. [26] produced a $10 \mu \mathrm{m}$-period magnetic lattice of 1000 1D traps formed from a perpendicularly magnetised $1 \mu \mathrm{m}$-thick TbGdFeCo film on a grooved silicon substrate on an atom chip plus bias fields (Fig. 1(b)). About $10^{8}{ }^{87} \mathrm{Rb}$ atoms were initially trapped in a mirror magneto-optical trap (MOT) and then confined in a compressed MOT using the quadrupole field from a current-carrying U-wire plus bias field. Atoms in the $\left|F=2, m_{F}=+2\right\rangle$ low field-seeking state were then transferred to a $\mathrm{Z}$-wire magnetic trap where they were RF evaporatively cooled to $\sim 15 \mu \mathrm{K}[60]$. 


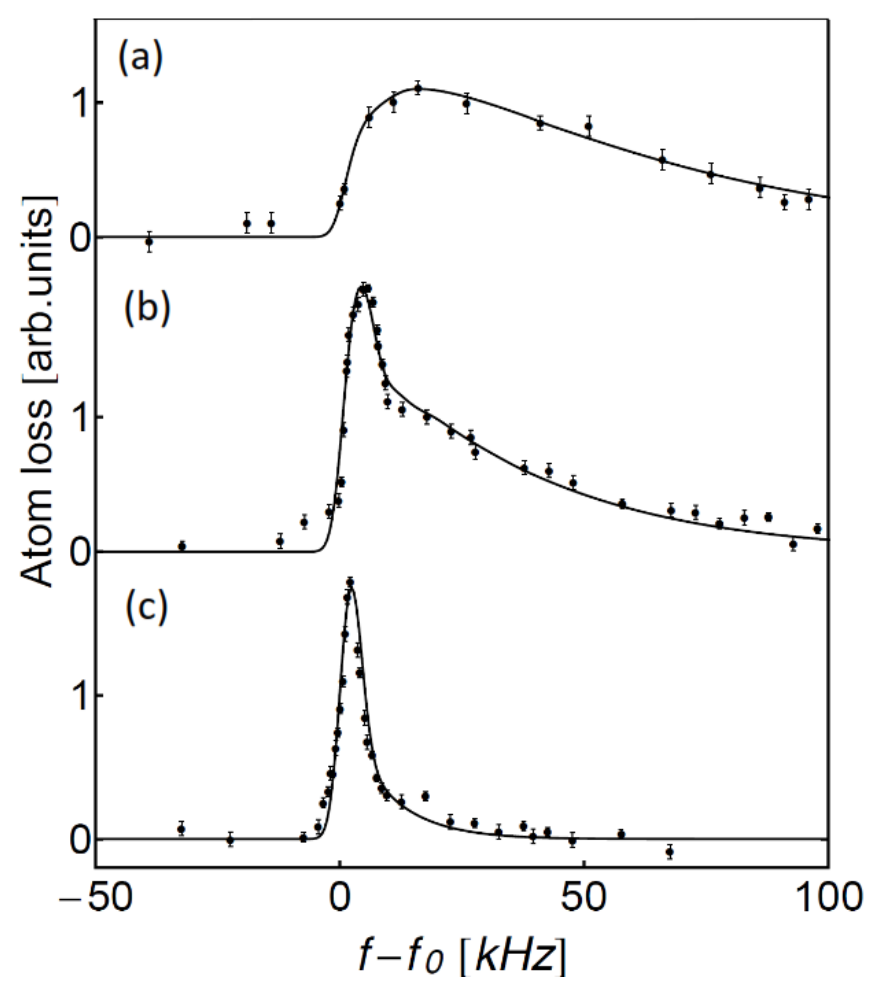

Fig. 2 Radiofrequency spectra of ${ }^{87} \mathrm{Rb}\left|F=1, m_{F}=-1\right\rangle$ atoms in one of the $\sim 100$ atom clouds trapped in a $10 \mu \mathrm{m}$-period 1D magnetic lattice, demonstrating the onset of Bose-Einstein condensation with increased evaporative cooling. The solid lines are fits to the data points based on a self-consistent mean-field model for a BEC plus thermal cloud as described in the text. The trap depths $\delta f=f_{f}-f_{0}$, temperatures and atom numbers obtained from this analysis are (a) $600 \mathrm{kHz}, 2.0 \mu \mathrm{K}, 5350$ atoms, (b) $400 \mathrm{kHz}, 1.3 \mu \mathrm{K}, 3430$ atoms and (c) $100 \mathrm{kHz}, 0.38 \mu \mathrm{K}, 200$ atoms. Adapted from [35]

The atoms in the Z-wire trap were then brought close $(\sim 5 \mu \mathrm{m})$ to the chip surface by ramping down the Z-wire current $\left(I_{\mathrm{z}}\right)$ and ramping up the bias field $B_{\text {by }}$ to $30 \mathrm{G}$ to create the magnetic lattice microtraps. When the Z-wire trap merged with the magnetic lattice traps, $I_{z}$ was reduced to zero with $B_{\mathrm{b} x}=B_{\min }=15 \mathrm{G}$. In this way, typically $3 \times$ $10^{6}$ atoms were loaded into $\sim 100$ magnetic lattice traps in the central region of the lattice, with barrier heights $\sim 1 \mathrm{mK}$ and trap frequencies in the range $\omega_{\text {rad }} / 2 \pi=20-90 \mathrm{kHz}$, $\omega_{a x} / 2 \pi \approx 1 \mathrm{~Hz}$. Radiofrequency spectroscopy measurements indicated temperatures $>150 \mu \mathrm{K}$, which were limited largely by the weak axial confinement that prevented efficient evaporative cooling in the lattice.

\section{Bose-Einstein condensation in multiple magnetic lattice sites}

A significant breakthrough was made in recent experiments by Jose et al. [33] and Surendran et al. [35] using the above magnetic lattice chip. The ${ }^{87} \mathrm{Rb}$ atoms were optically pumped into the $\left|F=1, m_{F}=-1\right\rangle$ low field-seeking state to minimise losses due to three-body recombination in the tightly confining magnetic traps [61, 62] and additional axial confinement was applied, with trap frequencies in the range $\omega_{\text {rad }} / 2 \pi=1.5-$ $20 \mathrm{kHz}, \omega_{a x} / 2 \pi=260 \mathrm{~Hz}$. Figure 2 shows radio- frequency spectra recorded for one of the $\sim 100$ atom clouds trapped in the magnetic lattice as the atoms were evaporatively cooled to lower trap depths $\delta f=f_{f}-f_{0}$ and lower temperatures (where $f_{f}$ is the final evaporation frequency and $f_{0}$ is the trap bottom). The spectra show the evolution from a broad thermal cloud distribution (Fig. 2(a)) to a bimodal distribution characteristic of a thermal cloud plus a narrow BEC distribution (Fig. 2(b)) to an almost pure BEC distribution (Fig. 2(c)) as the atom clouds are cooled through the critical temperature $(1.6 \mu \mathrm{K}$ for an ideal gas with $N=3000$ atoms).

The fits to the data points in Fig. 2 are based on a selfconsistent mean-field model for a BEC plus thermal cloud convolved with a Gaussian magnetic noise function (FWHM=4.3 kHz) [35]. The model includes the repulsive interaction among atoms in the BEC and in the thermal cloud and the mutual interaction between them but neglects the kinetic energy of the condensate atoms via the Thomas-Fermi approximation and the effects of gravity sag in the tight magnetic traps. The fits provide measurements of the trap bottom $\left(f_{0}\right)$, atom temperature $(T)$, condensate fraction $\left(N_{\mathrm{C}} / N\right)$ and chemical potential $(\mu)$. The effect of temperature is to change both the width of the broad thermal cloud component and the fraction of atoms in the condensate. 

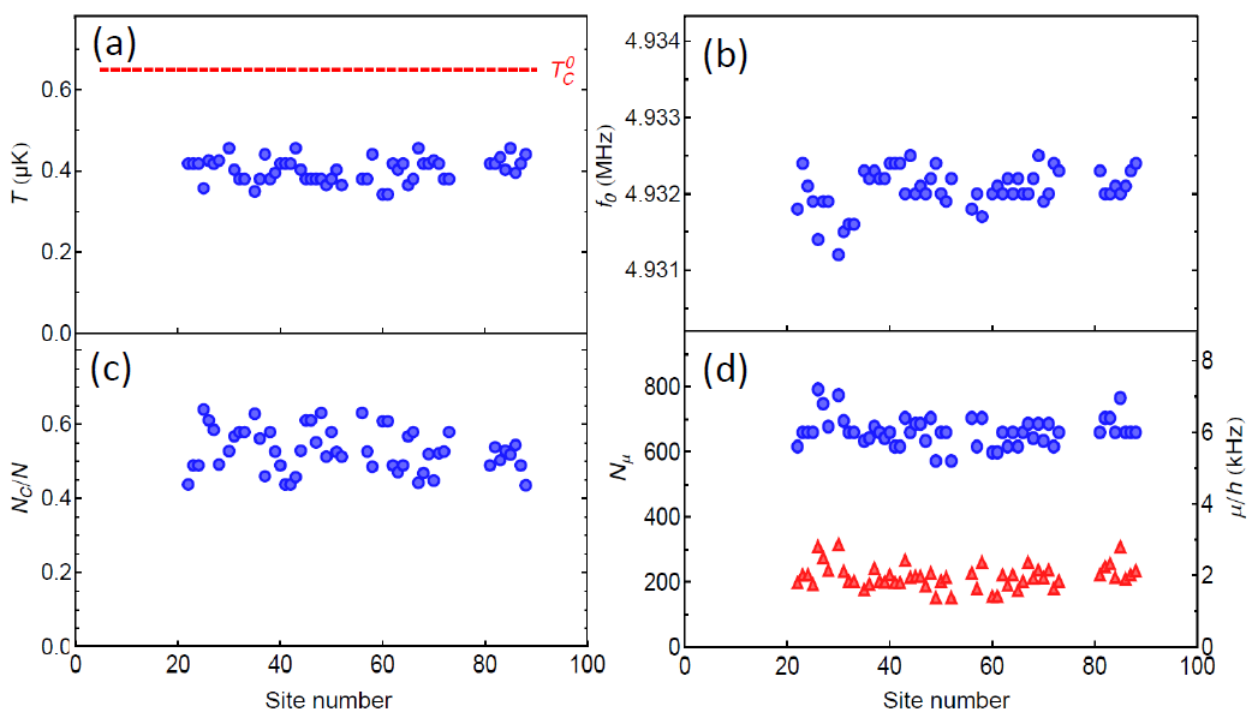

Fig. 3 (a) Atom temperature $T$, (b) trap bottom $f_{0}$, (c) condensate fraction $N_{\mathrm{C}} / N$ and (d) chemical potential $\mu$ (blue circles) and atom number $N_{\mu}$ derived from $\mu$ (red triangles), determined from fits to the RF spectra for multiple sites across the central region of the magnetic lattice, with trap depth $\delta f=100 \mathrm{kHz}, \omega_{\text {rad }} / 2 \pi=7.5 \mathrm{kHz}$. The red dashed line in (a) represents the ideal-gas critical temperature for 220 atoms. Adapted from [35]

Radiofrequency spectra taken simultaneously for all atom clouds across the central region of the magnetic lattice showed similar bimodal distributions to Fig. 2 with site-to-site variations in the above quantities that were consistent with the measurement errors (Fig. 3). In particular, the trap bottom $f_{0}$, which could be determined precisely from measurements of the frequency
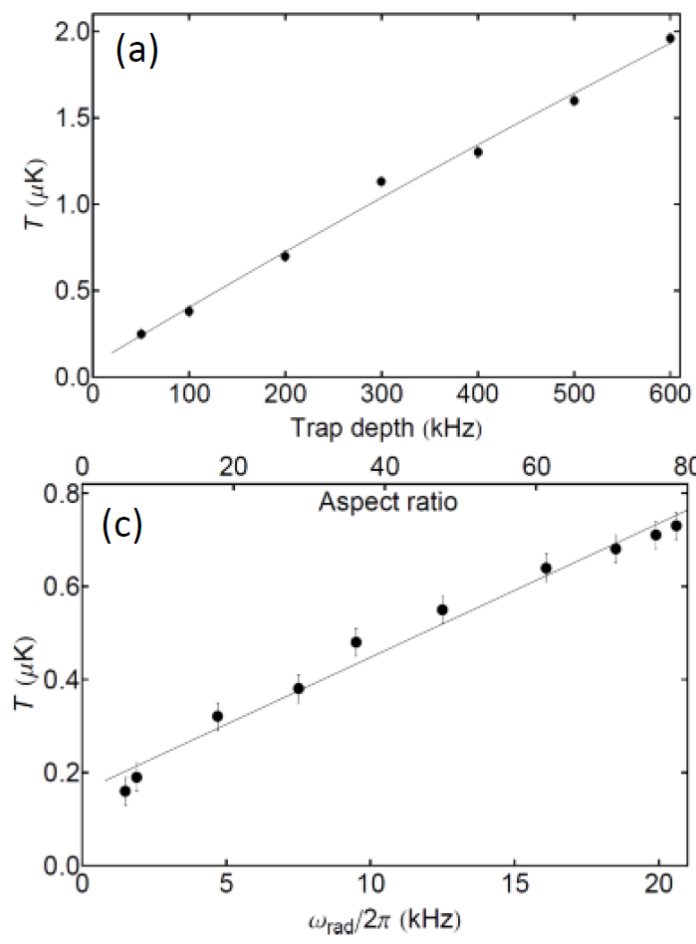

at which there were no atoms lost (Fig. 2(c)), showed onesigma variations of only $\pm 0.3 \mathrm{kHz}$ (or $\pm 0.4 \mathrm{mG}$ ) in $5 \mathrm{MHz}$ (Fig. 3(b)), reflecting the high uniformity in the central region of the magnetic lattice. The atom temperature is well below the ideal-gas critical temperature for all sites (Fig. 3(a)) and large condensate fractions are observed for all sites (Fig. 3(c)).
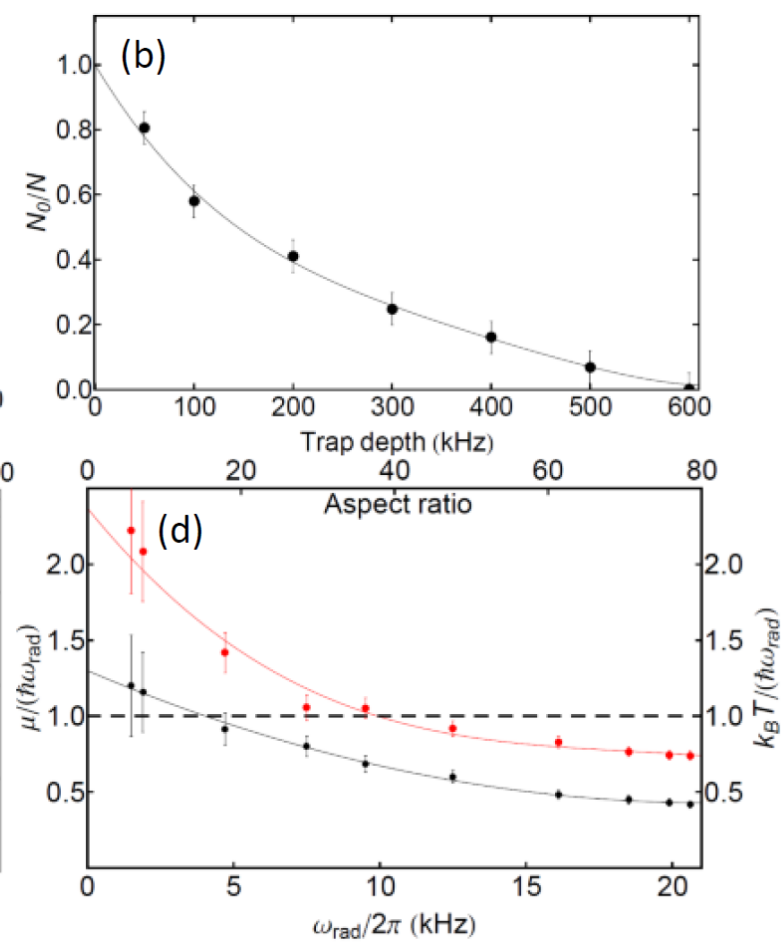

Fig. 4 Variation of (a) atom temperature $T$ and (b) condensate fraction $N_{\mathrm{C}} / N$ with trap depth $\delta f$ at trap frequencies $\omega_{\text {rad }} / 2 \pi=7.5$ $\mathrm{kHz}, \omega_{a x} / 2 \pi=260 \mathrm{~Hz}$; and variation of (c) atom temperature and (d) $\mu / \hbar \omega_{\text {rad }}$ (black points) and $k_{B} T / \hbar \omega_{\text {rad }}$ (red points) with radial trap frequency $\omega_{\text {rad }}$ at $\delta f=100 \mathrm{kHz}$. The horizontal dashed line in $(\mathbf{d})$ corresponds to $k_{\mathrm{B}} T=\mu=\hbar \omega_{\mathrm{rad}}$, which represents the energy of the lowest radial vibrational excited state. Adapted from [35] 


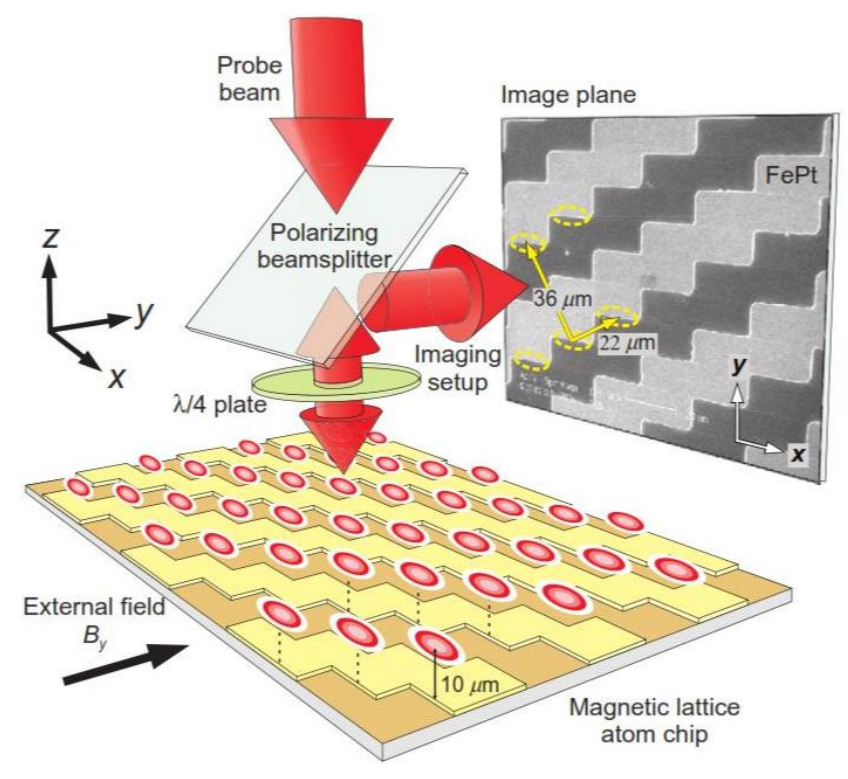

Fig. 5 Schematic of the absorption imaging of atom clouds trapped in a two-dimensional rectangular magnetic lattice. Reproduced from Whitlock et al. [27] with the permission of IOP Publishing

Figure 4 shows the variation of atom temperature $T$ and condensate fraction $N_{\mathrm{C}} / N$ with trap depth $\delta f$ and radial trap frequency $\omega_{\text {rad }} / 2 \pi$. At the lowest radial trap frequency $(1.5 \mathrm{kHz})$ a temperature of $0.16 \mu \mathrm{K}$ is achieved in the magnetic lattice (Fig. 4(c)) and at the smallest trap depth (50 kHz) a condensate fraction of $\sim 80 \%$ is observed (Fig. 4(b)). For radial trap frequencies $\omega_{\text {rad }} / 2 \pi>10 \mathrm{kHz}$, both the chemical potential $\mu$ and the thermal energy $k_{\mathrm{B}} T$ become smaller than the energy of the lowest radial vibrational excited state $\hbar \omega_{\text {rad }}$ (Fig. 4(d)), which represents the quasi-one-dimensional Bose gas regime [15, 63-67]. In Fig. 4(c) the atom temperatures determined from our analysis are seen to increase with radial trap frequency which is provisionally attributed to reduced efficiency of evaporative cooling due to suppression of rethermalising collisions in the quasi-1D regime [65].

Our one-dimensional magnetic lattice is able to create a large number of almost equivalent, highly elongated atom clouds. Thus, these lattices seem well suited for studying one-dimensional quantum gases. Using realistic parameters, e.g., $\omega_{\text {rad }} / 2 \pi=50 \mathrm{kHz}, \omega_{a x} / 2 \pi=100 \mathrm{~Hz}, N$ $=50$ atoms and $T=50 \mathrm{nK}$, it should be possible to reach the strongly interacting Tonks-Girardeau regime, which requires the Lieb-Liniger interaction parameter $\gamma \approx 2 a_{s} /\left(n_{1 \mathrm{D}} l_{\mathrm{rad}}^{2}\right)>1$ and the temperature parameter $t=$ $2 \hbar^{2} k_{B} T /\left(m g^{2}\right)<1 \quad[64]$. Here, $n_{1 \mathrm{D}}=N / l_{\mathrm{ax}}, l_{\mathrm{rad}}=$ $\sqrt{\hbar /\left(m \omega_{\text {rad }}\right)}, g \approx 2 \hbar \omega_{\text {rad }} a_{s}$ and $a_{s}$ is the s-wave scattering length.

\section{Two-dimensional magnetic lattices}

For many of the applications for which magnetic lattices are attractive, such as the quantum simulation of condensed matter phenomena and quantum information processing, two-dimensional lattices are usually required.
In 2007 Gerritsma et al. [25] created a two-dimensional rectangular magnetic lattice using a patterned, perpendicularly magnetised $\mathrm{FePt}$ magnetic film with periods of $36 \mu \mathrm{m}$ and $22 \mu \mathrm{m}$ in the $x, y$ directions plus bias fields. Whitlock et al. [27] extended this work to load and image individual clouds of ${ }^{87} \mathrm{Rb}\left|F=2, m_{F}=+2\right\rangle$ atoms in over 500 sites of the magnetic lattice. Figure 5 shows a schematic of the absorption imaging of the atom clouds trapped in the two-dimensional rectangular magnetic lattice. Losses due to rapid three-body recombination of the ${ }^{87} \mathrm{Rb}\left|F=2, m_{F}=+2\right\rangle$ atoms during evaporative cooling in the tight traps prevented the formation of BoseEinstein condensates with an observable condensate fraction.

One of the challenges with designing a two-dimensional magnetic lattice, especially lattices with high symmetry such as a square lattice [32], is the occurrence of magnetic field zeros, which lead to Majorana spin flips and loss of atoms. In 2010 Schmied et al. [29] developed a numerical algorithm for designing optimised magnetic microstructures to create periodic arrays of microtraps of various geometries with non-zero magnetic field minima. Figures 6(a) and (b) show magnetic film patterns designed to create square and triangular magnetic lattices at a trapping height $z_{\min }=a / 2$. The corresponding magnetic potentials are shown in Figs. 6(c) and (d). The magnetic film patterns are equivalent to those produced by an electric current passing around the perimeter of the film pattern, which for the square magnetic lattice has a similar shape to that of a square array of $Z$-wires (which have non-zero magnetic field minima). Schemes for loading the ultracold atoms from a Z-wire trap into a square or triangular magnetic lattice are given in [29, 68]

In 2014 Leung et al. [34] used the above algorithm to design square and triangular magnetic lattices with period $10 \mu \mathrm{m}$, and successfully loaded ${ }^{87} \mathrm{Rb}\left|F=2, m_{F}=+2\right\rangle$ atoms into both lattices at temperatures of about $35 \mu \mathrm{K}$ (Fig. 7). 
(a)

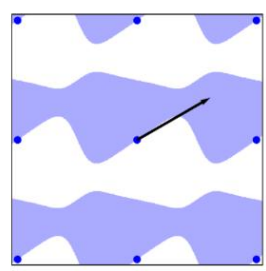

(c)

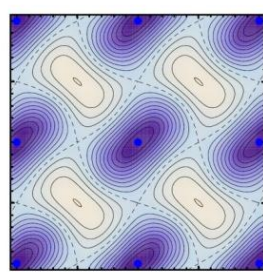

(b)

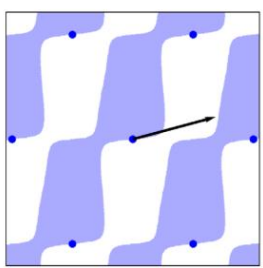

(d)

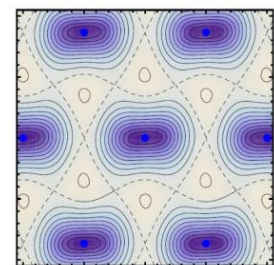

Fig. 6 Magnetic film patterns to create (a) square and (b) triangular magnetic lattices at a trapping height $z$ min $=a / 2$ [29]. Blue regions represent the magnetic film. The black dots indicate the positions of the magnetic field minima and the black arrows show the direction of the magnetic field at the minima. The resulting magnetic potentials are shown in (c) and (d), where the blue regions represent the potential minima. Reproduced from Schmied et al. [29] with the permission of IOP Publishing.

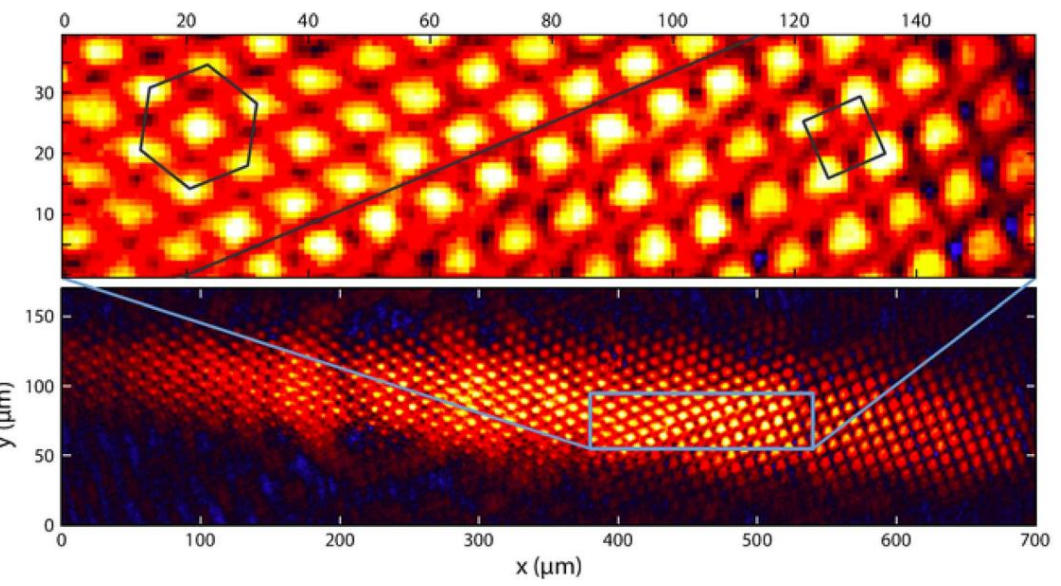

Fig. 7 Absorption images of ${ }^{87} \mathrm{Rb}$ atoms loaded into triangular (left side) and square (right side) magnetic lattices with period $10 \mu \mathrm{m}$. The lattice sites contain $\sim 360$ and $\sim 440$ atoms for the square and triangular lattices, respectively. The upper image is a magnified view of the central region. Reproduced from Leung et al. [34] with the permission of AIP Publishing.

\section{Sub-micron period magnetic lattices}

To date, one-dimensional magnetic lattices [26, 30, 33, 35] and two-dimensional rectangular [25, 27], square and triangular [34] magnetic lattices have been produced to trap periodic arrays of ultracold atoms with periods down to 10 $\mu \mathrm{m}$. For these lattice periods and for realistic barrier heights, there can be no quantum tunnelling of atoms between lattice sites, and the arrays of atoms represent isolated clouds with no interaction between them. To achieve significant tunnelling, lattice spacings of less than $1 \mu \mathrm{m}$ are required.

Herrera et al. [36] have recently reported the fabrication and characterisation of square and triangular magnetic lattice structures with a period of $0.7 \mu \mathrm{m}$. For $a=0.7 \mu \mathrm{m}$ and barrier height $V_{0} \sim 12 E_{R}$ (where $E_{R}=h^{2} / 8 m a^{2}$ is the lattice recoil energy), the tunnelling rate is estimated to be $J \sim 17 \mathrm{~Hz}$ [8]. This rate is compatible with the estimated lifetimes of the trapped atom clouds in these lattices (see below) and is suitable for realising the superfluid to Mott insulator transition, demonstrating the accessibility of magnetic lattices to the Hubbard model.

The magnetic microstructures were fabricated by patterning a $\mathrm{Co} / \mathrm{Pd}$ multi-atomic layer film ( 8 bi-layers of $0.28 \mathrm{~nm} \mathrm{Co}+0.9 \mathrm{~nm} \mathrm{Pd})[36,69]$ on a silicon substrate using electron-beam lithography followed by reactive ion etching. Multi-atomic layer $\mathrm{Co} / \mathrm{Pd}$ film was chosen because of its strong perpendicular magnetic anisotropy and very small grain size ( $6 \mathrm{~nm}$ [70] compared with $\sim 40 \mathrm{~nm}$ for TbGdFeCo [71]), allowing smooth and welldefined magnetic potentials at very small periods [72], as well as its high remanent magnetisation $(5.9 \mathrm{kG})$ and coercivity (1.0 kOe) [36]. 
(a)

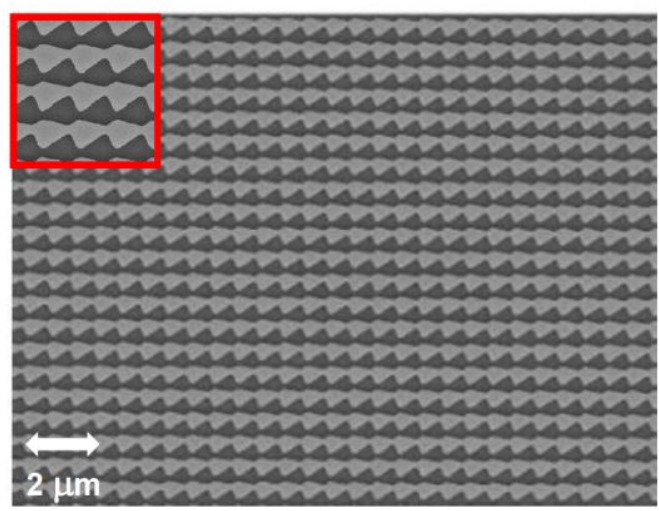

(b)

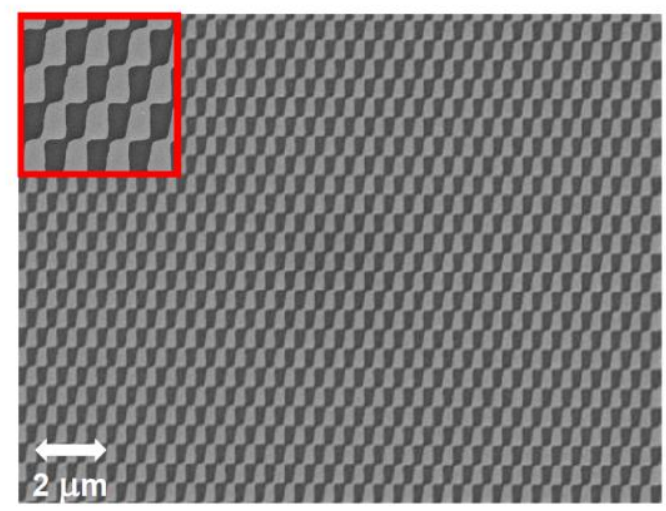

Fig. 8 SEM images of the fabricated Co/Pd magnetic microstructures to create (a) square and (b) triangular magnetic lattices with period $0.7 \mu \mathrm{m}$. The light grey regions represent the (unetched) magnetic film. The insets show part of the images magnified

Figure 8 shows SEM images of the $0.7 \mu \mathrm{m}$-period square and triangular magnetic microstructures, which illustrate the quality of the structures. The magnetised microstructures were characterised using magnetic force microscopy and magneto-optical Kerr effect [36].

Our simulations indicate that the square magnetic lattice with magnetisation $4 \pi M_{\mathrm{z}}=5.9 \mathrm{kG}$, magnetic film thickness $t=2.2 \mathrm{~nm}$ and bias fields $B_{\mathrm{b} x}=1.7 \mathrm{G}, B_{\mathrm{b} y}=$ $-0.8 \mathrm{G}$ create cylindrically symmetric traps at $z_{\mathrm{min}}=$ $0.35 \mu \mathrm{m}$ from the magnetic film surface, with the long axis in the [110] direction (Fig. 6(c)). For these parameters the trap minima are $B_{\min }=1.1 \mathrm{G}$, the barrier heights are $\Delta B_{y, x}$ $=1.4 \mathrm{G}$ (or $47 \mu \mathrm{K}$ for $\left.F=1, m_{F}=-1\right), \Delta B_{z}=0.8 \mathrm{G}$ (or $26 \mu \mathrm{K}$ ), and the trap frequencies perpendicular and parallel to the long axis are $\omega_{\perp} / 2 \pi=\omega_{z} / 2 \pi=120 \mathrm{kHz}, \omega_{\|} / 2 \pi=$ $37 \mathrm{kHz}$. Comparable results are obtained for the triangular lattice [36]. Larger barrier heights may be achieved by using thicker $\mathrm{Co} / \mathrm{Pd}$ magnetic films.

Previous studies have shown that the trap lifetime of an atom cloud may be significantly shortened as the cloud approaches within a few microns of a surface [73-75]. At these distances the attractive van der Waals and CasimirPolder forces between the atoms and the surface can distort the magnetic potential so that it is no longer trapping. For atoms located $0.3 \mu \mathrm{m}$ from the surface of a $0.7 \mu \mathrm{m}$-period magnetic microstructure, we estimate a critical trapping frequency at which the magnetic traps begin to fold to be $\omega_{\text {crit }} / 2 \pi \approx 44 \mathrm{kHz}$ [36], which is smaller than the trapping frequency $\omega_{z} / 2 \pi=120 \mathrm{kHz}$. In the case of a microstructured magnetic surface, the attractive van der Waals and Casimir-Polder forces may be swamped by the exponentially increasing repulsive magnetic force close to the surface. The trap lifetime may also be shortened by Johnson magnetic noise which arises from random thermal currents flowing in a conducting surface film that can induce spin flips and loss of atoms [73, 76-78]. For ${ }^{87} \mathrm{Rb}$ $\left|F=1, m_{F}=-1\right\rangle$ atoms trapped $0.3 \mu \mathrm{m}$ from a $50 \mathrm{~nm}-$ thick gold conducting film at $T=300 \mathrm{~K}$, we estimate a thermal spin-flip lifetime of $\tau_{\mathrm{Au}} \approx 180 \mathrm{~ms}$ [36], which is longer than the estimated tunnelling time of $60 \mathrm{~ms}$ for a $0.7 \mu \mathrm{m}$-period lattice with barrier height $V_{0} \sim 12 E_{\mathrm{R}}$ (20 $\mathrm{mG}$ ), or $13 \mathrm{~ms}$ for $V_{0} \sim 6 E_{\mathrm{R}}$. The spin-flip lifetime may be lengthened by using a reflecting film with higher resistivity such as palladium for which $\tau_{\mathrm{Pd}} \approx 870 \mathrm{~ms}$.

\section{Long-range interacting Rydberg atoms in a magnetic lattice}

An alternative approach for creating interaction between atoms on neighbouring sites of a magnetic lattice is to utilise long-range interacting Rydberg atoms [31, 34, 68, 79]. Highly excited Rydberg atoms can be orders of magnitude larger than ground-state atoms, making them very sensitive to fields and to one another. At large separations the interaction between Rydberg atoms is largely due to van der Waals interactions, scaling as $C_{6} / r^{6}$, where $C_{6}$ scales with principal quantum number as $n^{11}$. Each magnetic lattice site is prepared with one Rydberg atom in an ensemble of ground-state atoms via 'Rydberg blockade', in which the presence of the Rydberg atom shifts the energy levels of nearby atoms, suppressing subsequent excitation of other atoms in the ensemble [80]. The characteristic range of the Rydberg-Rydberg interaction is given by the blockade radius, $r_{b} \approx\left|C_{6} / \Omega\right|$, which for a typical atom-light coupling constant $\Omega$ / $2 \pi \sim 1 \mathrm{MHz}$ is $5-10 \mu \mathrm{m}$ (depending on the Rydberg state). To prepare a single Rydberg atom in an ensemble of spatial extent $l$ on each site of a lattice of period $a$ requires $l \ll r_{b} \leq a$, which can be met for a large spacing ( $a \sim$ $10 \mu \mathrm{m})$ magnetic lattice.

A potential issue when using long-range interacting Rydberg atoms in magnetic lattices is the effect of stray electric fields [79, 81-83]. During each cooling and trapping sequence $\mathrm{Rb}$ atoms can stick to the surface of the atom chip to create inhomogeneous electric fields [84] that can perturb the nearby Rydberg atoms. Studies by Tauschinsky et al. [79] of Rb Rydberg atoms trapped at distances down to $20 \mu \mathrm{m}$ from a gold-coated chip surface have revealed small distance-dependent energy shifts of $\sim \pm 10 \mathrm{MHz}$ for $n \approx 30$. Recent studies have demonstrated that the stray electric fields can be effectively screened out by depositing a uniform film of $\mathrm{Rb}$ over the entire gold surface [81] or by using a smooth monocrystalline quartz surface film coated with a monolayer of $\mathrm{Rb}$ adsorbates [82]. More studies are required to understand these effects. 


\section{$7 \quad$ Summary and perspectives}

Significant advances have recently been made in the development of magnetic lattices based on patterned magnetic films as a complementary tool to optical lattices for trapping periodic arrays of ultracold atoms and degenerate quantum gases.

Trapping of ultracold atoms in a one-dimensional magnetic lattice $[26,30]$ and two-dimensional rectangular $[25,27]$, square and triangular magnetic lattices [34] with periods down to $10 \mu \mathrm{m}$ has been demonstrated. Using a recently developed numerical algorithm [29] magnetic lattices based on patterned magnetic films may now, in principle, be tailored with nearly arbitrary configurations and lattice spacings. In the future, it should be possible to produce complex 2D geometries, such as honeycomb and kagome lattices and superlattices. Magnetic lattices based on patterned magnetic films on an atom chip are compact, robust and permanent, making them suitable for incorporating into devices, such as 'atomtronic' circuits [47].

Bose-Einstein condensation has been achieved in multiple sites of a magnetic lattice of one-dimensional microtraps with period $10 \mu \mathrm{m}[33,35]$. High condensate fractions $(\sim 80 \%)$, low atom temperatures $(\sim 0.16 \mu \mathrm{K})$ and a high degree of lattice uniformity have been demonstrated in a magnetic lattice.

For the magnetic lattices produced to date, which have periods $\geq 10 \mu \mathrm{m}$, the arrays of ultracold atoms represent isolated clouds with no interaction between the atoms on neighbouring sites. To enable quantum tunnelling of atoms between sites, lattices with sub-micron periods are required. High quality square and triangular magnetic lattice structures with periods of $0.7 \mu \mathrm{m}$ have recently been fabricated by patterning a $\mathrm{Co} / \mathrm{Pd}$ multi-atomic layer magnetic film [36]. These magnetic lattices would allow the quantum simulation of condensed matter systems such as the Hubbard model [5]. In the future, it should be possible to load fermionic atoms, such as ${ }^{40} \mathrm{~K}$, into magnetic lattices to simulate electrons in condensed matter systems such as graphene-like systems.

Another scheme to create interaction between lattice sites is to utilise long-range interacting Rydberg atoms in a large spacing $(\sim 10 \mu \mathrm{m})$ magnetic lattice [31]. These magnetic lattices would allow the quantum simulation of spin models such as the Heisenberg spin model including anisotropic or beyond nearest neighbour spin-spin interactions between Rydberg atoms [85].

Acknowledgments This work is supported by an Australian Research Council Discovery Project grant (DP130101160). We thank M. Singh for his contributions to the early stages of our experiments; M. Albrecht and D. Nissen from the University of Augsburg for providing the Co/Pd magnetic films; and A. Balcytis, P. Michaux and S. Juodkazis for fabricating the magnetic microstructures. We thank the Institute of Physics Publishing for permission to reproduce Figs. 1(b), 5 and 6 and the American Institute of Physics Publishing for permission to reproduce Fig. 7.
Conflict of interest The authors declare that they have no conflict of interest.

\section{References}

1. Chu S (1998) Nobel lecture: the manipulation of neutral particles. Rev Mod Phys 70: 685-706

2. Cohen-Tannoudji CN (1998) Nobel lecture: manipulating atoms with photons. Rev Mod Phys 70: 707-719

3. Phillips WD (1998) Nobel lecture: laser cooling and trapping of neutral atoms. Rev Mod Phys 70: 721-741

4. Morsch O, Oberthaler M (2006) Dynamics of Bose-Einstein condensates in optical lattices. Rev Mod Phys 78: 179-215

5. Lewenstein M, Sanpera A, Ahufinger V, Damski B, Sen(De) A, Sen U (2007) Ultracold atomic gases in optical lattices: mimicking condensed matter physics and beyond. Adv Phys 56: 243-379

6. Bloch I, Dalibard J, Zwerger W (2008) Many-body physics with ultracold gases. Rev Mod Phys 80: 885-964

7. Takamoto M, Hong F-L, Higashi R, Katori H (2005) An optical lattice clock. Nature 435: 321-324

8. Bakr WS, Gillen JI, Peng A, Fölling S, Greiner M (2009) A quantum gas microscope for detecting single atoms in a BoseHubbard regime optical lattice. Nature 462: 74-77

9. Calarco T, Hinds EA, Jaksch D, Schmiedmayer J, Cirac J I, Zoller P (2000) Quantum gates with neutral atoms: controlling collisional interactions in time-dependent traps. Phys Rev A 61: 022304

10. Monroe C (2002) Quantum information processing with atoms and photons. Nature 416: 238-246

11. Greiner M, Mandel O, Esslinger T, Hänsch TW, Bloch I (2002) Quantum phase transition from a superfluid to a Mott insulator in a gas of ultracold atoms. Nature 415: 39-44

12. Uehlinger T, Jotzu G, Messer M, Greif D, Hoffstetter W, Bissbort U, Esslinger T (2013) Artificial graphene with tunable interactions. Phys Rev Lett 111: 185307

13. Simon J, Bakr WS, Ma R, Tai ME, Preiss PM, Greiner M (2011) Quantum simulation of antiferromagnetic spin chains in an optical lattice. Nature 472: 307-312

14. Hart RA, Duarte PM, Yang T-L, Liu X, Paiva T, Khatami E, Scalettar RT, Trivedi N, Huse DA, Hulet RG (2015) Observation of antiferromagnetic correlations in the Hubbard model with ultracold atoms. Nature 519: 211-214

15. Kinoshita T, Wenger T, Weiss DS (2006) A quantum Newton's cradle. Nature 440: 900-903

16. Martiyanov K, Makhalov V, Turlapov A (2010) Observation of a two-dimensional Fermi gas of atoms. Phys Rev Lett 105: 030404

17. Billy J, Josse V, Zuo Z, Bernard A, Hambrecht B, Lugan P, Clement D, Sanchez-Palencia L, Bouyer P, Aspect A (2008) Direct observation of Anderson localization of matter waves in a controlled disorder. Nature 453: 891-894

18. Roati G, D'Errico C, Fallani L, Fattori M, Fort C, Zaccanti M, Modugno G, Modugno M, Inguscio M (2008) Anderson localization of a non-interacting Bose-Einstein condensate. Nature 453: 895-898

19. Mancini M, Pagano G, Cappellini G, Livi L, Rider M, Catani J, Sias C, Zoller P, Inguscio M, Dalmonte M, Fallani L (2015) Observation of chiral edge states with neutral fermions in synthetic Hall ribbons. Science 349: 510-1513

20. Cataliotti FS, Burger S, Fort C, Maddaloni P, Minardi F, Trombettoni A, Smerzi A, Inguscio M (2001) Josephson junction arrays with Bose-Einstein condensates. Science 293: 843-846

21. Hinds EA, Hughes I G (1999) Magnetic atom optics: mirrors, guides, traps, and chips for atoms. J Phys D 32: R119-R146 
22. Ghanbari S, Kieu TD, Sidorov A, Hannaford P (2006) Permanent magnetic lattices for ultracold atoms and quantum degenerate gases. J Phys B 39: 847-890

23. Gerritsma R, Spreeuw RJC (2006) Topological constraints on magnetostatic traps. Phys Rev A 74: 043405

24. Ghanbari S, Kieu TD, Hannaford P (2007) A class of permanent magnetic lattices for ultracold atoms. J Phys B 40: 1283-1294

25. Gerritsma R, Whitlock S, Fernholz T, Schlatter H, Luigjes JA, Thiele J-U, Goedkoop JB, Spreeuw RJC (2007) Lattice of microtraps for ultracold atoms based on patterned magnetic films. Phys Rev A 76: 033408

26. Singh M, Volk M, Akulshin A, Sidorov A, McLean R, Hannaford P (2008) One dimensional lattice of permanent magnetic microtraps for ultracold atoms on an atom chip. J Phys B 41: 065301

27. Whitlock S, Gerritsma R, Fernholz T, Spreeuw RJC (2009) Two-dimensional array of microtraps with atomic shift register on a chip. New J Phys 11: 023021

28. Abdelrahman A, Vasilev M, Alameh K, Hannaford P (2010) Asymmetrical two-dimensional magnetic lattices for ultracold atoms. Phys Rev A 82: 012320

29. Schmied R, Leibfried D, Spreeuw RJC, Whitlock S (2010) Optimized magnetic lattices for ultracold atomic ensembles. New J Phys 12: 103029

30. Llorente Garcia I, Darquie B, Curtis EA, Sinclair CDJ, Hinds EA (2010) Experiments on a videotape atom chip: fragmentation and transport studies. New J Phys 12: 093017

31. Leung VYF, Tauschinsky A, van Druten NJ, Spreeuw RJC (2011) Microtrap arrays on magnetic film atom chips for quantum information science. Quant Inf Process 10: 955-974

32. Ghanbari S, Abdelrahman A, Sidorov A, Hannaford P (2014) Analysis of a simple square magnetic lattice for ultracold atoms. J Phys B 47: 115301

33. Jose S, Surendran P, Wang Y, Hererra I, Krzemien L, Whitlock S, McLean R, Sidorov A, Hannaford P (2014) Periodic array of Bose-Einstein condensates in a magnetic lattice. Phys Rev A 89: 051602(R)

34. Leung VYF, Pijn DRM, Schlatter H, Torralbo-Campo L, La Rooij AL, Mulder G B, Naber J, Soudijn ML, Tauschinsky A, Abarbanel C, Hadad B, Golan E, Folman R, Spreeuw RJC (2014) Magnetic-film atom chip with $10 \mu \mathrm{m}$ period lattices of microtraps for quantum information science with Rydberg atoms. Rev Sci Instrum 85: 053102

35. Surendran P, Jose S, Wang Y, Hererra I, Hu H, Liu X, Whitlock S, McLean R, Sidorov A, Hannaford P (2015) Radiofrequency spectroscopy of a linear array of BoseEinstein condensates in a magnetic lattice. Phys Rev A 91: 023605

36. Herrera I, Wang Y, Michaux P, Nisson D, Surendran P, Juodkazis S, Whitlock S, McLean R, Sidorov A, Albrecht M, Hannaford P (2015) Sub-micron period lattice structures of magnetic microtraps for ultracold atoms on an atom chip. $\mathrm{J}$ Phys D 48: 115002

37. Yin J, Gao W, Hu J, Wang Y (2002) Magnetic surface microtraps for realizing an array of alkali atomic BoseEinstein condensates or Bose clusters. Opt Commun 206: 99113

38. Grabowski A, Pfau T (2003) A lattice of magneto-optical and magnetic traps for cold atoms. Eur Phys J D 22: 347-354

39. Günther A, Kraft S, Kemmler M, Koelle D, Kleiner R, Zimmermann C, Fortágh J (2005) Diffraction of a BoseEinstein condensate from a magnetic lattice on a microchip. Phys Rev Lett 95: 170405

40. Yun M, Yin J (2006) Practical scheme to realize 2D array of BECs on an atom chip: novel 2D magneto-optical and magnetic lattices. Opt Express 14: 2539-2551
41. West AD, Weatherill KJ, Hayward TJ, Fry PW, Schrefl T, Gibbs, MRJ, Adams CS, Allwood DA, Hughes IG (2012) Realization of the manipulation of ultracold atoms with a reconfigurable nonmagnetic system of domain walls. Nano Letters 12: 4065-4069

42. Romero-Isart O, Navau C, Sanchez A, Zoller P, Cirac JI (2013) Superconducting vortex lattices for ultracold atoms. Phys. Rev. Lett.111: 145304

43. Luo X, Wu L, Chen J, Lu R, Wang R, You L (2015) Generating an effective magnetic lattice for ultracold atoms. New J. Phys. 17: 083048

44. Yu J, Xu Z-F, Lu R, You L (2016) Dynamical generation of topological magnetic lattices for ultracold atoms. Phys. Rev. Lett. 116: 143003

45. Whitlock S, Hall BV, Roach T, Anderson R, Volk M, Hannaford P, Sidorov AI (2007) Effect of magnetization inhomogeneity on magnetic microtraps for atoms. Phys Rev A 75: 043602

46. Fernholz T, Gerritsma R, Whitlock S, Barb I, Spreeuw RJC (2010) Fully permanent magnet atom chip for Bose-Einstein condensation. Phys Rev A 77: 033409

47. Pepino RA, Cooper J, Meiser D, Anderson DZ, Holland MJ (2010) Open quantum systems approach to atomtronics. Phys Rev A 82: 013640

48. Sidorov A, Hannaford P (2011) From magnetic mirrors to atom chips. In Reichel J, Vuletic V (eds) Atom chips, WileyVCH, New York, Ch 1, pp 3-31

49. Opat GI, Wark SJ, Cimmino A (1992) Electric and magnetic mirrors and gratings for slowly moving neutral atoms and molecules. Appl Phys B 54: 396-402

50. Roach TM, Abele H, Boshier MG, Grossman HL, Zetie KP, Hinds EA (1995) Realization of a magnetic mirror for cold atoms. Phys Rev Lett 75: 629-632

51. Sidorov AI, McLean RJ, Rowlands WJ, Lau DC, Murphy JE, Walkiewicz M, Opat GI, Hannaford P (1996) Specular reflection of cold caesium atoms from a magnetostatic mirror. Quant Semiclass Opt. 8: 713-725

52. Sidorov AI, McLean RJ, Scharnberg F, Gough DS, Davis TJ, Sexton BA, Opat GI, Hannaford P (2002) Permanent magnet microstructures for atom optics. Acta Phys Polonica B 33: 2137-2155

53. Lau DC, Sidorov AI, Opat GI, McLean RJ, Rowlands WJ, Hannaford P (1999) Reflection of cold atoms from an array of current-carrying conductors. Eur J Phys D 5: 193-199

54. Lau DC, McLean RJ, Sidorov AI, Gough DS, Koperski J, Rowlands WJ, Sexton BA, Opat GI, Hannaford P (1999) Magnetic mirrors with micron-scale periodicities for slowly moving neutral atoms. J Opt. B 1: 371-377

55. Drndić M, Zabow G, Lee CS, Thywissen JH, Johnson KS, Prentiss M, Westervelt RM, Featonby PD, Savalli V, Cognet L, Helmerson K, Westbrook N, Westbrook CI, Phillips WD, Aspect A (1999) Properties of electromagnet mirrors as reflectors of cold Rb atoms. Phys Rev A 60: 4012-4015

56. Sinclair CDJ, Curtis EA, Llorente Garcia I, Retter JA, Hall BV, Eriksson S, Sauer BE, Hinds EA (2005) Bose-Einstein condensation on a permanent-magnet atom chip. Phys Rev A 72: 031603(R)

57. Boyd M, Streed EW, Medley P, Campbell GK, Mun J, Ketterle W, Pritchard DE (2007) Atom trapping with a thin magnetic film. Phys Rev A 76: 043624

58. Surendran P (2014) Bose-Einstein condensation in a magnetic lattice. $\mathrm{PhD}$ thesis, Swinburne University of Technology

59. Singh M (2008) A magnetic lattice and macroscopic entanglement of a BEC on an atom chip. PhD thesis, Swinburne University of Technology 
60. Hall BV, Whitlock S, Scharnberg F, Hannaford P, Sidorov A (2006) A permanent magnetic film atom chip for Bose-Einstein condensation. J Phys B 39: 27-36

61. Burt E, Ghrist RW, Myatt CJ, Holland MJ, Cornell EA, Wieman CE (1997) Coherence, correlations, and collisions: what one learns about Bose-Einstein condensates from their decay. Phys Rev Lett 79: 337-340

62. Söding J, Guéry-Odelin D, Desbiolles P, Chevy F, Inamori H, Dalibard J (1999) Three-body decay rate of a rubidium BoseEinstein condensate. Appl Phys B 69: 257-261

63. Görlitz A, Vogels JM, Leanhardt AE, Raman C, Gustavson TL, Abo-Shaeer JR, Chikkatur AP, Gupta S, Inouye S, Rosenband T, and Ketterle W (2001) Realization of BoseEinstein condensates in lower dimensions. Phys Rev Lett 87 130402

64. Greiner M, Bloch I, Mandel O, W. Hänsch TW, Esslinger T (2001) Exploring phase coherence in a 2D lattice of BoseEinstein condensates. Phys Rev Lett 87160405

65. Masets IE, Schmiedmayer J (2010) Thermalization in a quasi1D ultracold bosonic gas. New J Phys 12: 055023

66. Jacqmin T, Armijo J, Berrada T, Kheruntsyan KV and Bouchoule I (2011) Sub-Poisonnian fluctuations in a 1D Bose gas: from the quantum quasicondensate to the strongly interacting regime. Phys Rev Lett 106: 230405

65. Moritz H, Stöferle T, Köhl M, Esslinger T (2003) Exciting collective oscillations in a trapped 1D gas. Phys Rev Lett 91: 250402

68. Tauschinsky A (2013) Rydberg atoms on a chip and in a cell. $\mathrm{PhD}$ thesis, University of Amsterdam

69. Stärk M, Schlickeiser F, Nissen D, Hebler B, Graus P, Hinzke D, Scheer E, Leiderer P, Fonin M, Albrecht M, Nowak U, Boneberg J (2015) Controlling the magnetic structure of $\mathrm{Co} / \mathrm{Pd}$ thin films by direct laser interference patterning. Nanotechnology 26: 205302

70. Roy AG, Laughlin DE, Klemmer TJ, Howard K, Khizroev S, Litvinov D (2001) Seed layer effect on microstructure and magnetic properties of Co/Pd multilayers. J Appl Phys 89: 7531-7533

71. Wang JY, Whitlock S, Scharnberg F, Gough DS, Sidorov AI, McLean RJ and Hannaford P (2005) Perpendicularly magnetized, grooved $\mathrm{GdTbFeCo}$ microstructures for atom optics. J Phys D 38: 4015-4020

72. Robertson N, Magnetic data storage with patterned media www.nnin.org/doc/snmr10/Cornell_nanomanuf_2010.pdf
73. Harber DM, McGuirk JM, Obrecht JM, Cornell EA (2003) Thermally induced losses in ultra-cold atoms magnetically trapped near room-temperature surfaces. J Low Temp Phys 133: $229-238$

74. Lin YJ, Teper I, Chin C, Vuletic V (2004) Impact of CasimirPolder potential and Johnson noise on Bose-Einstein condensate stability near surfaces. Phys Rev Lett 92: 050404

75. Treutlein P (2008) Coherent manipulation of ultracold atoms on atom chips. $\mathrm{PhD}$ dissertation, Ludwig Maximilian University of Munich

76. Henkel C, Pötting S, Wilkens M (1999) Loss and heating of particles in small and noisy traps. Appl Phys B 69379

77. Jones MPA, Vale CV, Sahagun D, Hall BV, Hinds EA (2003) Spin coupling between cold atoms and the thermal fluctuations of a metal surface. Phys Rev Lett 91: 080401

78. Rekdal PK, Scheel S, Knight PL, Hinds EA (2004) Thermal spin flips in atom chips. Phys Rev A 70: 013811

79. Tauschinsky A, Thijssen RMT, Whitlock S, van Linden van den Heuvell HB, Spreeuw RJC (2010) Spatially resolved excitation of Rydberg atoms and surface effects on an atom chip. Phys Rev A 81: 063411

80. Saffman M, Walker T, Molmer K (2010) Quantum information with Rydberg atoms. Rev Mod Phys 82: 23132363

81. Hermann-Avigliano C, Teixeira RC, Nguyen TL, CantatMoltrecht T, Nogues G, Dotsenko I, Gleyzes S, Raimond JM, Haroche S, Brune M (2014) Long coherence times for Rydberg qubits on a superconducting atom chip. Phys Rev A 90: 040502

82. Sedlacek JA, Kim E, Rittenhouse ST, Weck PF, Sadeghpour SR, Shaffer JP (2016) Electric field cancellation on quartz by $\mathrm{Rb}$ absorbate-induced negative electron affinity. Phys Rev Lett 116: 133201

83. Naber J, Machluf S, Torralbo-Campo L, Soudijn ML, van Druten NJ, van Linden van den Heuvell HB, Spreeuw RJC (2015) Absorbate dynamics on a silica-coated gold surface measured by Rydberg Stark spectroscopy. arXiv:1512.07511

84. McGuirk JM, Harber DM, Obrecht JM, Cornell EA (2004) Alkali-metal adsorbate polarization on conducting and insulating surfaces probed with Bose-Einstein condensates. Phys Rev A 69: 062905

85. Weimer H, Müller M, Büchler HP, Lesanovsky I (2011) Digital quantum simulation with Rydberg atoms. Quant Inf Process 10: 885-906 